\title{
Prognostic significance of serum alkaline phosphatase and lactate dehydrogenase levels in patients with unresectable advanced gastric cancer
}

\author{
Tsutomu Namikawa ${ }^{1}$. Nobuko Ishida ${ }^{1}$. Sachi Tsuda ${ }^{1} \cdot K_{\text {Kazune Fujisawa }}{ }^{*}$ Eri Munekage ${ }^{1}$. Jun Iwabu ${ }^{1}$. \\ Masaya Munekage ${ }^{1}$. Sunao Uemura ${ }^{1}$. Shigehiro Tsujii ${ }^{1}$ - Takahiko Tamura ${ }^{2} \cdot$ Tomoaki Yatabe $^{2} \cdot$ Hiromichi Maeda $^{3}$. \\ Hiroyuki Kitagawa ${ }^{1} \cdot$ Michiya Kobayashi $^{3,4} \cdot$ Kazuhiro Hanazaki $^{1}$
}

Received: 22 August 2018 / Accepted: 6 November 2018 / Published online: 11 November 2018

(c) The International Gastric Cancer Association and The Japanese Gastric Cancer Association 2018

\begin{abstract}
Background This study evaluated the prognostic value of alkaline phosphatase (ALP) and lactate dehydrogenase (LDH) together with host-related factors in patients with unresectable advanced gastric cancer.

Methods The study enrolled 262 patients who received chemotherapy for unresectable advanced gastric cancer at Kochi Medical School from 2007 to 2015. Clinicopathological information and systemic inflammatory response data were analyzed for associations between baseline cancer-related prognostic variables and survival outcomes.

Results The median survival time was significantly lower for patients with high ALP, high LDH, high total bilirubin, high aspartate aminotransferase, high alanine transaminase, high gamma-glutamyltransferase, high creatinine, a Glasgow prognostic score (GPS) of 1 or 2 score compared to GPS 0, higher compared to lower neutrophil to lymphocyte ratio (NLR) 3.9, lower compared to higher prognostic nutrition index 36.1, T3-4 compared to T1-2 tumor and diffuse-type compared to intestinaltype histology. Multivariate survival analysis identified high ALP 322 (HR 1.808; 95\% CI 1.015-3.220; $P=0.044$ ), T2-3 (HR 2.622; 95\% CI 1.224-5.618; $P=0.013$ ), and diffuse-type gastric cancer (HR 2.325; 95\% CI 1.341-4.032; $P=0.003$ ) as significant independent predictors of worse prognosis in the studied group of cancer patients.

Conclusions High level of ALP is an independent, worse prognosis factor for patients receiving chemotherapy for unresectable and recurrent gastric cancer.
\end{abstract}

Keywords Alkaline phosphatase $\cdot$ Lactate dehydrogenase $\cdot$ Gastric cancer $\cdot$ Prognostic value $\cdot$ Survival

\section{Introduction}

Gastric cancer is a common malignant tumor that ranks seventh in leading causes of cancer mortality worldwide and second for cancer-related deaths in Japan [1, 2]. While

Tsutomu Namikawa

tsutomun@kochi-u.ac.jp

1 Department of Surgery, Kochi Medical School, Kohasu, Oko-cho, Nankoku, Kochi 783-8505, Japan

2 Department of Anesthesiology and Intensive Care Medicine, Kochi Medical School, Kochi, Japan

3 Cancer Treatment Center, Kochi Medical School Hospital, Kochi, Japan

4 Department of Human Health and Medical Sciences, Kochi Medical School, Kochi, Japan extensive surgery with regional lymphadenectomy is an effective treatment for localized disease, chemotherapy with or without molecular targeted drugs is the recommended treatment for patients with unresectable advanced or metastatic gastric cancer [3].

The transformation of normal cells to cancer cells or the proliferation of cancer cells always leads to abnormal serum enzyme synthesis, sometimes before changes in tumor morphology are evident, that is, before disease is clinically detectable [4]. Alkaline phosphatase (ALP) in human serum can be secreted from liver, bone, small intestine, placenta, and a wide variety of tumors $[4,5]$, while lactate dehydrogenase (LDH), a key enzyme for the conversion of pyruvate to lactate during glycolysis, is required for tumor maintenance [6]. In cancer cells, anaerobic glycolysis is the main metabolic pathway for energy production. Both ALP and LDH 
might, therefore, serve as useful blood indicators for tumor detection and monitoring.

Recently, some investigators reported that ALP and LDH appeared to be reliable parameters to predict prognosis for various malignancies such as pancreatic cancer or esophageal squamous cell carcinoma [7, 8]. However, the influence of serum enzymes such as ALP and LDH has not been reported in gastric cancer patients with regard to prognosis. On the other hand, the systemic inflammatory response represented by the Glasgow prognostic score (GPS) or neutrophil to lymphocyte ratio (NLR) is quite valuable because it can be easily and inexpensively measured by blood sampling as well as routinely tested serum enzymes including ALP and LDH [9].

Cancer-related inflammation affects the tumor microenvironment modulation system. In the process of tumor development, cancer may cause central necrosis due to rapid growth, by which LDH is induced and inflammation has occurred. In this process, increased systemic inflammatory response may reflect enzyme induction by liver tissue or increased cellular permeability. We, therefore, sought to explore such a relationship by investigating the prognostic value of ALP and LDH levels in serum together with wellestablished tumor-related factors such as systemic inflammatory response markers in patients receiving chemotherapy for unresectable advanced gastric cancer.

\section{Materials and methods}

\section{Patients}

Two hundred and sixty-two patients with unresectable advanced or recurrent gastric cancer who were treated with chemotherapy at Kochi Medical School during the period from January 2007 to December 2015 were identified from a medical information database. Gastric cancer diagnoses were determined by esophagogastroduodenoscopy, biopsy specimen analysis, computed tomography, magnetic resonance imaging, ultrasonography of the abdomen, and positron emission tomography.

The patients' demographic data and clinicopathological characteristics including histologic type, depth of tumor invasion, and lymph node metastasis were retrospectively collected from our institution's database collection. Patients with a history of medical treatment for any disease were considered to have a comorbidity, which information was obtained from medical history records or during the pretreatment workup such as blood test, pulmonary function tests, electrocardiography and chest radiography. Blood samples were collected and analyzed for serum concentrations of albumin, ALP, LDH, total bilirubin, aspartate aminotransferase (AST), alanine transaminase (ALT), gamma-glutamyltransferase $(\gamma \mathrm{GT})$, creatinine and C-reactive protein (CRP), as well as neutrophil and lymphocyte cell counts. Tumor histology was categorized as intestinal type (well differentiated, moderately differentiated, and papillary adenocarcinoma) or diffuse type (poorly differentiated, mucinous adenocarcinoma and signet ring cell carcinoma) according to Lauren's classification.

Overall survival (OS) after treatment was recorded, with survival time defined as the interval between the date of chemotherapy initiation and the date of death or last contact.

\section{Measurement of serum variables}

Venous blood samples were taken at the time of diagnosis and during chemotherapy, and the percentages of particular cell types were determined in Giemsa-stained blood smears. Cutoff values for the target serum variables were defined by the upper limit of normal (ULN) values set by the automatic biochemical detector used in our hospital for biochemical analysis, at $322 \mathrm{U} / \mathrm{L}$ for ALP, $222 \mathrm{U} / \mathrm{L}$ for $\mathrm{LDH}, 1.5 \mathrm{mg} / \mathrm{dL}$ for total bilirubin, $30 \mathrm{U} / \mathrm{L}$ for AST, $42 \mathrm{U} / \mathrm{L}$ for ALT, $64 \mathrm{U} / \mathrm{L}$ for $\gamma \mathrm{GT}, 1.07 \mathrm{mg} / \mathrm{dL}$ for creatinine, respectively. A serum enzyme level above the cutoff value was defined as an increased serum enzyme level. The neutrophil count divided by the lymphocyte count was recorded as NLR.

GPS and prognostic nutrition index (PNI) were constructed as previously described to evaluate inflammatory status prior to chemotherapy and thus determine which score is more reflective of prognosis in patients with gastric cancer [10-12].

\section{Statistical analysis}

We tested differences between mean values for the two patient groups for significance by the Mann-Whitney $U$ test for continuous variables and Pearson's Chi-square test for categorical variables. We used the Kaplan-Meier method to generate cumulative survival rates and compared them using the log-rank test to evaluate statistically significant differences. Statistical analyses were performed using SPSS for Windows, version 22.0. A multivariate Cox proportional hazards regression analysis was used to identify factors independently associated with survival. For the subgroup analysis of OS, the hazard ratio (HR) and $95 \%$ confidence interval (CI) within each subgroup were summarized. When the various factors were considered in a multivariate analysis, all were dichotomized according to the univariate analysis.

\section{Results}

\section{Patient characteristics}

The study cohort comprised 171 men and 91 women with a median age of 69 years (range 19-89 years). A total of 
153 patients $(58.4 \%)$ had comorbidities, which include renal dysfunction in 104 (39.7\%), hepatic disorder in 87 (33.2\%), cardiovascular diseases in $71(27.1 \%)$ and pulmonary diseases in 44 patients $(16.8 \%)$. The median ALP and LDH levels were $240 \mathrm{U} / \mathrm{L}$ (range 113-9029 U/L) and $198 \mathrm{U} / \mathrm{L}$ (range 105-3573 U/L), respectively.

Sixteen patients with both a human epidermal growth factor receptor 2 (HER2) status of $3+$ or $2+$ (based on immunohistochemical staining of tumor samples) and positive result by fluorescence in situ hybridization analyses following their clinical examination were treated with concomitant trastuzumab and chemotherapy comprising capecitabine plus cisplatin, according to the ToGA trial that validated the additive effects of trastuzumab for HER2-positive tumors [13]. The vast majority of patients were treated using platinum compounds plus fluoropyrimidines as first-line treatment according to recent large-scale randomized controlled trials $[14,15]$. The first-line chemotherapy treatment regimen was $\mathrm{S}-1$ plus cisplatin in $79, \mathrm{~S}-1$ alone in $76, \mathrm{~S}-1$ plus oxaliplatin in 47 , trastuzumab with capecitabine plus cisplatin in 31 , capecitabine plus cisplatin in 17 , ramucirumab plus paclitaxel in 6, capecitabine plus oxaliplatin in 2, docetaxel plus 5-fluorouracil and cisplatin in 2, S-1 plus ramucirumab in 2 patients. The median course of chemotherapy was 6 courses (range 1-18 courses). One hundred and seventyeight patients were shifted to second-line chemotherapy using taxanes or irinotecan after evidence of disease progression. The median survival time (MST) was 11.3 months (range 1.3-74.1 months) and the overall 1-, 2-, and 3-year survival rates after therapy were $47.2 \%, 25.0 \%$, and $16.0 \%$, respectively.

\section{Association of serum enzyme levels and comorbidities}

Serum ALP and LDH levels according to comorbidities of the patients with unresectable or recurrent gastric cancer are shown in Table 1. The median LDH level for the patients with liver or bone metastasis of gastric cancer was significantly higher than those without liver or bone metastasis ( $228 \mathrm{U} / \mathrm{L}$ vs. $185 \mathrm{U} / \mathrm{L} ; P<0.001)$. Similarly, median LDH level for the patients with hepatic disorders was significantly higher than those without hepatic disorders (219 U/L vs. $188 \mathrm{U} / \mathrm{L} ; P=0.007)$. Although median ALP level for the patient with liver or bone metastasis was higher than those without liver or bone metastasis ( $275 \mathrm{U} / \mathrm{L}$ vs. $233 \mathrm{U} / \mathrm{L})$, the difference was not statistically significant.

\section{Association of clinicopathological factors and survival}

Table 2 summarizes the clinical characteristics and survival of patients in the present study using univariate
Table 1 Serum alkaline phosphatase and lactate dehydrogenase levels according to comorbidities of patients with unresectable or recurrent gastric cancer

\begin{tabular}{|c|c|c|c|c|}
\hline \multirow[t]{2}{*}{ Characteristics } & \multicolumn{2}{|c|}{$\begin{array}{l}\text { Alkaline phos- } \\
\text { phatase }\end{array}$} & \multicolumn{2}{|c|}{$\begin{array}{l}\text { Lactate dehydroge- } \\
\text { nase }\end{array}$} \\
\hline & $\begin{array}{l}\text { Median } \\
\text { level } \\
(\mathrm{U} / \mathrm{L})\end{array}$ & $P$ value & $\begin{array}{l}\text { Median } \\
\text { level } \\
\text { (U/L) }\end{array}$ & $P$ value \\
\hline Liver or bone metastasis & & 0.052 & & $<0.001$ \\
\hline Positive & 275 & & 228 & \\
\hline Negative & 233 & & 185 & \\
\hline Viral hepatitis & & 0.534 & & 0.161 \\
\hline Positive & 240 & & 223 & \\
\hline Negative & 240 & & 197 & \\
\hline Hepatic disorders & & 0.077 & & 0.007 \\
\hline Positive & 266 & & 219 & \\
\hline Negative & 235 & & 188 & \\
\hline
\end{tabular}

analysis. The median, pretreatment serum levels of ALP and LDH across all patients $(n=262)$ were $240 \mathrm{U} / \mathrm{L}$ (range 113-9029) and $198 \mathrm{U} / \mathrm{L}$ (range 105-3573), respectively. The MST was significantly higher for patients with ALP $<322$ (machine cut-off value in U/L) than for those with ALP $\geq 322$ (11.8 months vs. 8.3 months; $P=0.010)$, as shown in Fig. 1. Similarly, the MST of patients with LDH $<222$ was significantly higher than of those with LDH $\geq 222$ (12.8 months vs. 7.3 months; $P=0.005$ ), as shown in Fig. 2. The MST was also significantly higher in the intestinal-type group than in the diffuse-type group (13.1 months vs. 9.2 months; $P=0.009$ ), as shown in Fig. 3. The MST of patients with T1 or T2 tumor was significantly higher than of those with $\mathrm{T} 3$ or $\mathrm{T} 4$ tumor (17.9 months vs. 11.1 months; $P=0.033$ ). Additionally, total bilirubin $\geq 1.5 \mathrm{mg} / \mathrm{dL}$, AST $\geq 30 \mathrm{U} / \mathrm{L}$, ALT $\geq 42 \mathrm{U} / \mathrm{L}$, $\gamma \mathrm{GT} \geq 64 \mathrm{U} / \mathrm{L}$ and creatinine $\geq 1.07 \mathrm{mg} / \mathrm{dL}$ were found to be significant prognostic factors according to the results of the univariate log-rank tests.

Based on the pretreatment median NLR across all 262 patients of 3.9 , patients were divided into two groups (i.e., NLR $<3.9$ and $\geq 3.9$ ). They were similarly divided for survival analysis based on the pretreatment median PNI of 36.1 (i.e., two groups of PNI $\geq 36.1$ and PNI <36.1). The MST was also significantly higher for patients with a GPS of zero compared to those with a GPS of 1 or 2 (15.3 months vs. 9.5 months; $P=0.005$ ), a lower NLR 3.9 than a higher NLR 3.9 (15.3 months vs. 9.5 months; $P<0.001$ ), and a PNI $\geq 36.1$ than a PNI $<36.1$ (13.1 months vs. 10.7 months; $P=0.040$ ). Age, gender, lymph node metastasis, disease status, and metastatic site had no significant association with survival rate by univariate analysis (Table 2). 
Table 2 Clinical characteristics and survival of patients with unresectable or recurrent gastric cancer using univariate analysis

\begin{tabular}{|c|c|c|c|c|c|}
\hline \multirow[t]{2}{*}{ Characteristics } & \multicolumn{3}{|c|}{ Survival rate (\%) } & \multirow{2}{*}{$\begin{array}{l}\text { Median survival } \\
\text { time (months) }\end{array}$} & \multirow[t]{2}{*}{$P$ value } \\
\hline & 1 year & 2 years & 3 years & & \\
\hline Overall & 47.2 & 25 & 16 & 11.3 & \\
\hline Age group (years) & & & & & 0.933 \\
\hline$<69$ & 45.4 & 24.4 & 12.1 & 10.8 & \\
\hline$\geq 69$ & 47 & 24.1 & 16.5 & 11.6 & \\
\hline Gender & & & & & 0.103 \\
\hline Male & 41.1 & 21.8 & 13.9 & 10.6 & \\
\hline Female & 58.9 & 29.8 & 13.7 & 15.6 & \\
\hline Histological type & & & & & 0.009 \\
\hline Intestinal type & 58 & 38.7 & 32.2 & 13.1 & \\
\hline Diffuse type & 41.3 & 18.5 & 11.1 & 9.2 & \\
\hline Tumor status & & & & & 0.033 \\
\hline $\mathrm{T} 1-2$ & 63.5 & 37.7 & 18.9 & 17.9 & \\
\hline $\mathrm{T} 2-3$ & 45.1 & 21.5 & 14.3 & 11.1 & \\
\hline Lymph node metastasis & & & & & 0.893 \\
\hline N0-1 & 48.0 & 29.2 & 12.8 & 10.6 & \\
\hline $\mathrm{N} 2-3$ & 46.3 & 21.9 & 14.5 & 11.5 & \\
\hline Disease status & & & & & 0.348 \\
\hline Initially metastatic & 45.3 & 22.7 & 14 & 10.9 & \\
\hline Recurrent after curative resection & 48.4 & 27.8 & 12.6 & 11.6 & \\
\hline Metastasis site & & & & & 0.252 \\
\hline Hematogenous metastasis (liver, lung, bone) & 48.8 & 25.2 & 7.8 & 11.6 & \\
\hline Peritoneum & 44.6 & 22.5 & 15.5 & 10.1 & \\
\hline Lymph node & 50.2 & 31.7 & 23.1 & 11.6 & \\
\hline $\operatorname{ALP}(\mathrm{U} / \mathrm{L})$ & & & & & 0.010 \\
\hline$<322$ & 50.3 & 27.3 & 19.9 & 11.8 & \\
\hline$\geq 322$ & 37.9 & 15.4 & 6.2 & 8.3 & \\
\hline LDH (U/L) & & & & & $<0.001$ \\
\hline$<222$ & 52 & 30.6 & 19.8 & 12.8 & \\
\hline$\geq 222$ & 33.1 & 8.8 & 4.4 & 7.3 & \\
\hline Total bilirubin & & & & & 0.012 \\
\hline$<1.5$ & 48.0 & 25.1 & 16.3 & 11.5 & \\
\hline$\geq 1.5$ & 0 & 0 & 0 & 6.7 & \\
\hline AST & & & & & 0.026 \\
\hline$<30$ & 51.6 & 26.9 & 17.1 & 12.3 & \\
\hline$\geq 30$ & 33.7 & 18.0 & 13.5 & 6.8 & \\
\hline ALT & & & & & 0.012 \\
\hline$<42$ & 49.6 & 25.9 & 16.4 & 11.6 & \\
\hline$\geq 42$ & 17.8 & 8.9 & 0 & 4.7 & \\
\hline$\gamma \mathrm{GT}$ & & & & & 0.006 \\
\hline$<64$ & 49.5 & 26.5 & 17.5 & 11.6 & \\
\hline$\geq 64$ & 29.9 & 6.2 & 0 & 7.1 & \\
\hline Creatinine & & & & & 0.027 \\
\hline$<1.07$ & 48.5 & 26.6 & 17.2 & 11.2 & \\
\hline$\geq 1.07$ & 27.8 & 0 & 0 & 6.8 & \\
\hline GPS & & & & & 0.005 \\
\hline 0 & 58.2 & 32.7 & 17.3 & 15.3 & \\
\hline 1 or 2 & 40.6 & 17.1 & 12.9 & 9.5 & \\
\hline NLR & & & & & $<0.001$ \\
\hline$<3.9$ & 55.9 & 29.4 & 15.5 & 15.3 & \\
\hline$\geq 3.9$ & 36.1 & 15.3 & 8.2 & 8.1 & \\
\hline PNI & & & & & 0.040 \\
\hline$\geq 36.1$ & 52.8 & 30.1 & 18.7 & 13.1 & \\
\hline$<36.1$ & 43.2 & 19.8 & 13.9 & 10.7 & \\
\hline
\end{tabular}


Table 2 (continued)

$A L P$ alkaline phosphatase, $L D H$ lactate dehydrogenase, $A S T$ aspartate aminotransferase, $A L T$ alanine transaminase, $\gamma G T$ gamma-glutamyltransferase, GPS Glasgow prognostic score, $N L R$ neutrophil to lymphocyte ratio, $P N I$ prognostic nutrition index

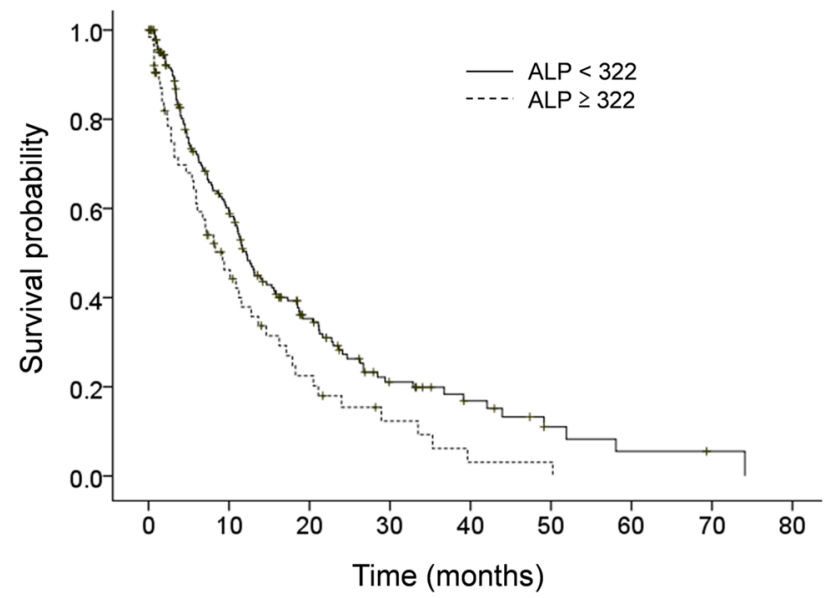

Fig. 1 Kaplan-Meier estimates of overall survival for patients with unresectable and recurrent gastric cancer in alkaline phosphatase $(\mathrm{ALP})<322$ (solid line) and ALP $\geq 322$ (dotted line) groups. There was a significant difference in survival between the groups $(P=0.010 ;$ stratified log-rank test $)$

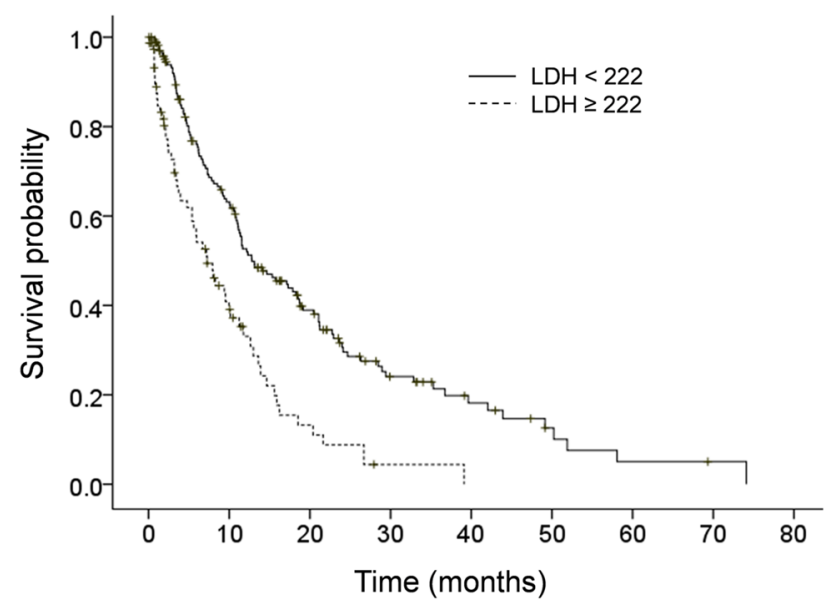

Fig. 2 Kaplan-Meier estimates of overall survival for patients with unresectable and recurrent gastric cancer in lactate dehydrogenase $(\mathrm{LDH})<222$ (solid line) and $\mathrm{LDH} \geq 222$ (dotted line) groups. There was a significant difference in survival between the groups $(P<0.001 ;$ stratified log-rank test $)$

\section{Multivariate survival analysis}

Comparing the survival rate among subgroups by univariate analysis identified the following potentially prognostic factors as significantly associated with a poor outcome: (1) diffuse type, (2) ALP $\geq 322$, (3) LDH $\geq 222$, (4) T3-4, (5) total bilirubin $\geq 1.5$, (6) AST $\geq 30$, (7) ALT $\geq 42$, (8) $\gamma \mathrm{GT} \geq 64$,

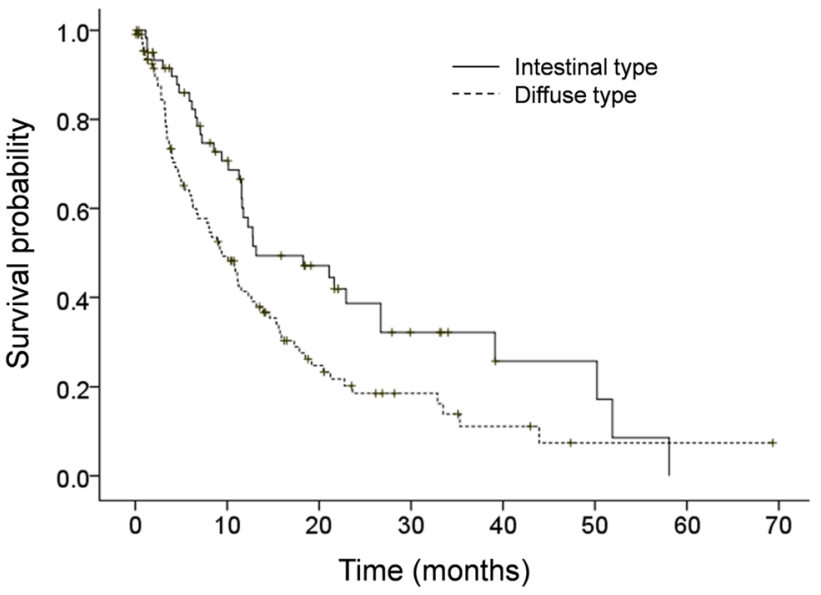

Fig. 3 Kaplan-Meier estimates of overall survival for patients with unresectable and recurrent gastric cancer in intestinal-type (solid line) and diffuse-type (dotted line) groups. There was a significant difference in survival between the groups $(P=0.021$; stratified log-rank test)

(9) creatinine $\geq 1.07$, (10) GPS 1 or 2 , (11) NLR $\geq 3.9$, and (12) PNI $<36.1$. By multivariate analysis, the following factors were found to be independently associated with poor survival: (1) diffuse-type gastric cancer (HR 2.325; 95\% CI 1.341-4.032; $P=0.003$ ), (2) ALP $\geq 322$ (HR $1.808 ; 95 \% \mathrm{CI}$ $1.015-3.220 ; P=0.044$ ), and (3) T3-4 (HR 2.622; 95\% CI 1.224-5.618; $P=0.013$ ), as shown in Table 3. LDH, total bilirubin, AST, ALT, $\gamma$ GT, creatinine, GPS, NLR, and PNI did not significantly influence survival rate.

\section{Discussion}

We found that high ALP levels independently predict poor prognosis in patients with unresectable advanced gastric cancer, indicating that such levels could be used to reflect clinical prognosis. These findings support previous studies that proposed ALP or LDH as prognostic markers in hepatocellular carcinoma, esophageal squamous cell carcinoma, nasopharyngeal carcinoma, and pancreatic carcinoma $[7,8$, 16-19]. This is the first study to demonstrate the relationship between ALP levels and prognosis in gastric cancer patients.

By multivariate analysis, ALP was independently related to poor survival in our patient cohort. ALP is a phosphate monoester hydrolase that catalyzes the hydrolysis and transfer of phosphate groups in alkaline conditions and is routinely assayed in clinical practice [20], based on strong evidence that tumors might secrete variant forms of ALP 
Table 3 Clinical characteristics and survival in patients with unresectable or recurrent gastric cancer using multivariate survival analysis

\begin{tabular}{llll}
\hline Variable & Hazard ratio & $95 \%$ confidence interval & $P$ value \\
\hline Histologic type (intestinal/diffuse) & 2.325 & $1.341-4.032$ & 0.003 \\
ALP $(<322 / \geq 322)$ & 1.808 & $1.015-3.220$ & 0.044 \\
LDH $(<222 / \geq 222)$ & 1.732 & $0.996-3.010$ & 0.052 \\
Tumor status (T1-2/T3-4) & 2.622 & $1.224-5.618$ & 0.013 \\
Total bilirubin $(<1.5 / \geq 1.5)$ & 3.404 & $0.897-12.917$ & 0.072 \\
AST $(<30 / \geq 30)$ & 0.929 & $0.496-1.738$ & 0.817 \\
ALT $(<42 / \geq 42)$ & 1.709 & $0.758-3.854$ & 0.196 \\
$\gamma$ GT $(<64 / \geq 64)$ & 0.763 & $0.314-1.851$ & 0.550 \\
Creatinine $(<1.07 / \geq 1.07)$ & 2.035 & $0.986-4.199$ & 0.055 \\
GPS $(0 / 1$ or 2$)$ & 0.865 & $0.412-1.814$ & 0.701 \\
NLR $(<3.9 / \geq 3.9)$ & 1.872 & $0.937-3.739$ & 0.076 \\
PNI $(\geq 36.1 /<36.1)$ & 1.490 & $0.728-3.049$ & 0.276 \\
\hline
\end{tabular}

$A L P$ alkaline phosphatase, $L D H$ lactate dehydrogenase, GPS Glasgow prognostic score, $N L R$ neutrophil to lymphocyte ratio, $P N I$ prognostic nutrition index into the circulation in detectable amounts [5, 21]. Specifically, Kojima et al. [22] reported significant changes in ALP activity in the liver of mice bearing Ehrlich ascites tumor, suggesting a link between the elevation of liver ALP activity and the proliferation of cancer cells. Although the exact mechanisms and function of LDH and ALP associated with tumor progression are still unclear, these enzymes could be tumor-associated antigens [23], although clinicians should also consider that ALP levels can be affected by metabolic disorders of bone disease and hepatic impairment.

A summary of previous reports demonstrating a relationship between ALP levels and cancer patient prognosis is presented in Table 4 . In these investigations, the primary lesion was hepatocellular carcinoma in two reports, with the remainder comprising nasopharyngeal carcinoma, esophageal cancer, and pancreatic cancer. Our cases comprised resectable cancer patients who underwent surgery in three studies and unresectable or recurrent cancer treated by chemotherapy, radiotherapy, or chemoradiotherapy in three studies. All studies confirmed that elevated ALP was associated with poor overall survival. In the present study, we used the cut-off value for ALP and LDH defined by the ULN values set by the machine used for biochemical analysis in our hospital. The majority of previous studies also used the ULN set in each hospital as the cut-off value, while $\mathrm{Xu}$ et al. [16] systematically explored the cut-off value of ALP, which is $121 \mathrm{U} / \mathrm{L}$, using the receiver operating characteristic (ROC) curve analysis to predict prognosis in 172 patients with hepatocellular carcinoma who underwent liver resection. Utilizing the ALP values with relatively high sensitivity and specificity in multivariate regression analysis, their results showed that ALP and tumor size were independent prognostic predictors of poor overall survival [16].

Tumor proliferation and development can cause metabolic changes in some serum enzymes, proteins, and hormones $[3,16]$. Despite the available oxygen levels, cancer cells preferentially use the anaerobic pathway of glycolysis to meet their energy requirements, a phenomenon historically known as the Warburg effect [24]. Anaerobic glycolysis substantially results in the conversion of pyruvate to lactate,

Table 4 Review of previous reports demonstrating a relationship between ALP levels and patient prognosis

\begin{tabular}{|c|c|c|c|c|c|c|}
\hline Author & Year & Primary lesion & Treatment & No. of cases & $\begin{array}{l}\mathrm{COL} \\
\text { of ALP } \\
(\mathrm{U} / \mathrm{L})\end{array}$ & Outcomes ( $\geq$ COL vs. $<$ COL $)$ \\
\hline Yu et al. [19] & 2011 & Hepatocellular carcinoma & Operation & 1685 & 82 & 5-Year OS rate, $35.8 \%$ vs. $56.2 \%$ \\
\hline Li et al. [17] & 2012 & Nasopharyngeal carcinoma & $\begin{array}{l}\text { Radiotherapy, chemo- } \\
\text { therapy, and chemoradio- } \\
\text { therapy }\end{array}$ & 533 & 110 & 5 -Year OS rate, $69 \%$ vs. $87 \%$ \\
\hline Xu et al. [16] & 2014 & Hepatocellular carcinoma & Operation & 172 & 121 & 5 -Year OS rate, $15.6 \%$ vs. $60.3 \%$ \\
\hline Wei et al. [7] & 2016 & Esophageal cancer & Chemotherapy & 906 & 90.7 & MST, 23.7 months vs. 45.4 months \\
\hline Ji et al. [8] & 2016 & Pancreatic cancer & Operation & 185 & 110 & 5 -Year OS rate, $0.0 \%$ vs. $8.0 \%$ \\
\hline Present series & 2018 & Gastric cancer & Chemotherapy & 262 & 322 & MST, 8.3 months vs. 11.8 months \\
\hline
\end{tabular}

$A L P$ alkaline phosphatase, $C O L$ cut-off level, $O S$ overall survival, $M S T$ median survival time 
and LDH catalyzes the final step in the glycolytic pathway, determining the production of adenosine triphosphate.

Although many diseases induce increases in LDH isoenzyme levels, including myocardial infarction and pulmonary embolism, LDH5 is most closely related to cancer [25]. Kolev et al. [25] reported that patients showing overexpression of LDH-5 had far lower disease-free (63.5\% vs. $82.7 \%)$ and overall (56.3\% vs. $78.4 \%)$ survival rates compared to patients with low LDH-5 expression among 152 patients with different grades and stages of gastric carcinoma. Furthermore, in mouse models, mitochondrial activity in tumor cells is affected by the knockdown of LDH-A, which encodes LDH5, resulting in lower tumorigenicity of malignant cells [6]. These results also indicate that targeting LDHA might provide a novel therapeutic strategy for cancer treatment via regulation of trigger activation pathways that control tumor growth and aggressiveness [17].

Our study findings also showed that diffuse-type gastric cancer was independently associated with poor survival compared to intestinal-type cancer. Previous studies also showed that histological type according to the Lauren classification had independent prognostic relevance to predicting the survival of postoperative patients with gastric cancer [1, $9,26]$, although some of these studies found no such independent association by multivariate analysis [27, 28]. Thus, the prognostic relevance of histological type according to the Lauren classification remains controversial, particularly because there are distinct tumor development pathways for intestinal and diffuse gastric cancer.

Previous studies demonstrated that the systemic inflammatory response-related factors including GPS, NLR and PNI were prognostic indicators in various cancers, because they reflect the tumor microenvironment, which are regarded as host-related factors induced by the tumor [9, 29-32]. Based on multivariate analysis in this study, we found that high ALP level is an independent factor to predict poor prognosis in advanced gastric cancer patients in addition to the traditional prognostic markers such as diffuse-type histology and further invaded tumor. Therefore, elevated ALP levels might predict prognosis of these patients with more precision than the systemic inflammatory response markers such as GPS, NLR, and PNI. One of the reasons of these results is that increased cellular permeability induced by systemic inflammation would be consistent with the correlation between albumin concentration and the activity of ALP which is primarily synthesized and function intracellularly [33]. In addition, the elevation of ALP could possibly reflect a heavy tumor burden, because certain subtypes of ALP have been found to be expressed in several malignant cell lines [7, 21, 23].

In the present study, LDH level was significantly higher in the patients with liver or bone metastasis and hepatic disorders than those without such situation. Similarly, ALP level showed the same tendency, although it was not significantly different. Accordingly, we should not be totally dependent on these markers to predict prognosis of the patients due to the regulation by hepatobiliary impairment or the metabolic disorders of bone disease including metastasis.

The present study has several potential limitations. First, selection bias may have influenced survival data due to the retrospective nature of this study. Second, this study was conducted in a single institution with a relatively small number of subjects, and thus could be affected by patient selection bias. Further studies with adequate statistical power and a larger number of patient subgroups are needed to determine the reliability and accuracy of using ALP and LDH during chemotherapy as a prognostic tool for advanced gastric cancer.

In conclusion, elevated ALP is associated with worse survival in patients with advanced gastric cancer, supporting these serum enzymes as useful biomarkers for prognosis prediction. Further studies are needed to confirm and extend our results to improve the management and treatment of advanced gastric cancer.

\section{Compliance with ethical standards}

Conflict of interest The authors declare that they have no conflict of interest.

\section{References}

1. Nashimoto A, Akazawa K, Isobe Y, Miyashiro I, Katai H, Kodera Y, et al. Gastric cancer treated in 2002 in Japan: 2009 annual report of the JGCA nationwide registry. Gastric Cancer. 2013;16:1-27.

2. Fidler MM, Gupta S, Soerjomataram I, Ferlay J, SteliarovaFoucher E, Bray F. Cancer incidence and mortality among young adults aged 20-39 years worldwide in 2012: a population-based study. Lancet Oncol. 2017;18:1579-89.

3. Ohtsu A, Yoshida S, Saijo N. Disparities in gastric cancer chemotherapy between the east and west. J Clin Oncol. 2006;24:2188-96.

4. Ferreira LM1, Hebrant A, Dumont JE. Metabolic reprogramming of the tumor. Oncogene. 2012;31:3999-4011.

5. Warnes TW, Timperley WR, Hine P, Kay G. Pancreatic alkaline phosphatase and a tumour variant. Gut. 1972;13:513-9.

6. Fantin VR, St-Pierre J, Leder P. Attenuation of LDH-A expression uncovers a link between glycolysis, mitochondrial physiology, and tumor maintenance. Cancer Cell. 2006;9:425-34.

7. Wei XL, Zhang DS, He MM, Jin Y, Wang DS, Zhou YX, et al. The predictive value of alkaline phosphatase and lactate dehydrogenase for overall survival in patients with esophageal squamous cell carcinoma. Tumour Biol. 2016;37:1879-87.

8. Ji F, Fu SJ, Guo ZY, Pang H, Ju WQ, Wang DP, et al. Prognostic value of combined preoperative lactate dehydrogenase and alkaline phosphatase levels in patients with resectable pancreatic ductal adenocarcinoma. Medicine (Baltimore). 2016;95:e4065.

9. Namikawa T, Munekage E, Munekage M, Maeda H, Yatabe $\mathrm{T}$, Kitagawa $\mathrm{H}$, et al. Evaluation of systemic inflammatory response biomarkers in patients receiving chemotherapy for 
unresectable and recurrent advanced gastric cancer. Oncology. 2016;90(6):321-6.

10. McMillan DC, Crozier JE, Canna K, Angerson WJ, McArdle CS. Evaluation of an inflammation-based prognostic score (GPS) in patients undergoing resection for colon and rectal cancer. Int $\mathbf{J}$ Colorectal Dis. 2007;22:881-6.

11. Wen J, Bedford M, Begum R, Mitchell H, Hodson J, Whiting $\mathrm{J}$, et al. The value of inflammation based prognostic scores in patients undergoing surgical resection for oesophageal and gastric carcinoma. J Surg Oncol. 2018;117(8):1697-707.

12. Tokunaga R, Sakamoto Y, Nakagawa S, Izumi D, Kosumi K, Taki $\mathrm{K}$, et al. Comparison of systemic inflammatory and nutritional scores in colorectal cancer patients who underwent potentially curative resection. Int J Clin Oncol. 2017;22:740-8.

13. Bang YJ, Van Cutsem E, Feyereislova A, Chung HC, Shen L, Sawaki A, et al. Trastuzumab in combination with chemotherapy versus chemotherapy alone for treatment of HER2-positive advanced gastric or gastro-oesophageal junction cancer (ToGA): a phase 3, open-label, randomised controlled trial. Lancet. 2010;376:687-97.

14. Koizumi W, Narahara H, Hara T, Takagane A, Akiya T, Takagi M, et al. S-1 plus cisplatin versus S-1 alone for first-line treatment of advanced gastric cancer (SPIRITS trial): a phase III trial. Lancet Oncol. 2008;9:215-21.

15. Boku N, Yamamoto S, Fukuda H, Shirao K, Doi T, Sawaki A, et al. Fluorouracil versus combination of irinotecan plus cisplatin versus $S-1$ in metastatic gastric cancer: a randomised phase 3 study. Lancet Oncol. 2009;10:1063-9.

16. Xu XS, Wan Y, Song SD, Chen W, Miao RC, Zhou YY, et al. Model based on $\gamma$-glutamyltransferase and alkaline phosphatase for hepatocellular carcinoma prognosis. World J Gastroenterol. 2014;20:10944-52.

17. Li G, Gao J, Tao YL, Xu BQ, Tu ZW, Liu ZG, et al. Increased pretreatment levels of serum LDH and ALP as poor prognostic factors for nasopharyngeal carcinoma. Chin J Cancer. 2012;31:197-206.

18. Faloppi L, Bianconi M, Giampieri R, Sobrero A, Labianca R, Ferrari $\mathrm{D}$, et al. The value of lactate dehydrogenase serum levels as a prognostic and predictive factor for advanced pancreatic cancer patients receiving sorafenib. Oncotarget. 2015;6:35087-94.

19. Yu MC, Chan KM, Lee CF, Lee YS, Eldeen FZ, Chou HS, et al. Alkaline phosphatase: does it have a role in predicting hepatocellular carcinoma recurrence? J Gastrointest Surg. 2011;15:1440-9.

20. Hirschhaeuser F, Sattler UG, Mueller-Klieser W. Metabolic key player in cancer. Cancer Res. 2011;71:6921-5.

21. Tokumitsu S, Tokumitsu K, Kohnoe K, Takeuchi T. Characterization of liver-type alkaline phosphatase from human gastric carcinoma cells (KMK-2) in vitro. Cancer Res. 1979;39:4732-8.
22. Kojima Y, Sakurada T. Increase in alkaline phosphatase activity in the liver of mice bearing Ehrlich ascites tumor. Cancer Res. 1976;36:23-7.

23. Dua P, Kang HS, Hong SM, Tsao MS, Kim S, Lee DK. Alkaline phosphatase ALPPL-2 is a novel pancreatic carcinoma-associated protein. Cancer Res. 2013;73(6):1934-45.

24. Warburg O. On the origin of cancer cells. Science. 1956;123:309-14.

25. Kolev Y, Uetake H, Takagi Y, Sugihara K. Lactate dehydrogenase-5 (LDH-5) expression in human gastric cancer: association with hypoxia-inducible factor (HIF-1alpha) pathway, angiogenic factors production and poor prognosis. Ann Surg Oncol. 2008; 15:2336-44.

26. Wang H, Xing XM, Ma LN, Liu L, Hao J, Feng LX, et al. Metastatic lymph node ratio and Lauren classification are independent prognostic markers for survival rates of patients with gastric cancer. Oncol Lett. 2018;15:8853-62.

27. Berlth F, Bollschweiler E, Drebber U, Hoelscher AH, Moenig S. Pathohistological classification systems in gastric cancer: diagnostic relevance and prognostic value. World J Gastroenterol. 2014;20(19):5679-84.

28. Mönig S, Baldus SE, Collet PH, Zirbes TK, Bollschweiler E, Thiele J, et al. Histological grading in gastric cancer by Goseki classification: correlation with histopathological subtypes and prognosis. Anticancer Res. 2001;21(1B):617-20.

29. Forrest LM, McMillan DC, McArdle CS, Angerson WJ, Dunlop DJ. Evaluation of cumulative prognostic scores based on the systemic inflammatory response in patients with inoperable nonsmall-cell lung cancer. Br J Cancer. 2003;89(6):1028-30.

30. Proctor MJ, Morrison DS, Talwar D, Balmer SM, O'Reilly DS, Foulis AK, et al. An inflammation-based prognostic score (mGPS) predicts cancer survival independent of tumour site: a Glasgow Inflammation Outcome Study. Br J Cancer. 2011;104(4):726-34.

31. Sugimoto K, Komiyama H, Kojima Y, Goto M, Tomiki Y, Sakamoto K. Glasgow prognostic score as a prognostic factor in patients undergoing curative surgery for colorectal cancer. Dig Surg. 2012;29(6):503-9.

32. Kitagawa H, Namikawa T, Munekage M, Fujisawa K, Kawanishi Y, Kobayashi M, et al. Preoperative patient-related factors associated with prognosis after esophagectomy for esophageal cancer. Esophagus. 2017;14(4):360-5.

33. Brown DJ, Milroy R, Preston T, McMillan DC. The relationship between an inflammation-based prognostic score (Glasgow Prognostic Score) and changes in serum biochemical variables in patients with advanced lung and gastrointestinal cancer. J Clin Pathol. 2007;60(6):705-8. 